\title{
ADAPTED LIST COLORING OF GRAPHS AND HYPERGRAPHS*
}

\author{
A. V. KOSTOCHKA ${ }^{\dagger}$ AND XUDING ZHU
}

\begin{abstract}
We introduce and study adapted list coloring of graphs and hypergraphs. This is a generalization of ordinary list coloring and adapted coloring, and has more applications than these. We prove that the upper bounds on the adaptable choosability of graphs and uniform hypergraphs in terms of maximum degree are sufficiently stronger than those on the ordinary choosability, while the bounds in terms of degeneracy are the same. We also characterize simple graphs with adaptable choosability 2 .
\end{abstract}

Key words. adapted coloring, list coloring, degree conditions

AMS subject classification. $05 \mathrm{C} 15$

DOI. $10.1137 / 070698385$

1. Introduction. Hell and Zhu [6] introduced and studied the notion of adapted coloring of graphs.

DeFINITION 1.1. Let $H$ be a multihypergraph (i.e., parallel hyperedges are allowed), and let $F: E(H) \rightarrow\{1,2, \ldots, k\}$ be an edge $k$-coloring of $H$ (not required to be proper). A vertex $k$-coloring $c: V \rightarrow\{1,2, \ldots, k\}$ of $H$ is adapted to $F$ if for every color $j$, the preimage $c^{-1}(j)$ does not contain any hyperedge of color $j$. In other words, if an edge e of $H$ has color $j$, then it cannot have all its vertices colored with $j$.

While the multiplicity of edges does not matter in the ordinary vertex coloring, it does in the adapted coloring. If every (hyper)edge of $G$ has multiplicity $k$ and $F$ colors these edges with all distinct $k$ colors, then any vertex $k$-coloring of $G$ adapted to this edge coloring is an ordinary proper $k$-coloring. But choosing different edge colorings yields different problems that have more applications than the ordinary vertex coloring. In particular, adaptable vertex coloring has applications to matrix partitions of graphs, trigraph homomorphisms, and full constraint satisfaction problems [3, 4, 5, 7].

The adaptable chromatic number is defined in a natural way.

DeFINITION 1.2. Let $G$ be a multihypergraph. The adaptable chromatic number $\chi_{a d}(G)$ of $G$ is the minimum integer $k$ such that for every edge $k$-coloring $F$ of $G$, there is a vertex $k$-coloring of $G$ adapted to $F$.

Hell and Zhu [6] proved that a nonempty graph $G$ has $\chi_{a d}(G) \leq 2$ if and only if $G$ has an edge $e$ such that $G-e$ is bipartite. Thus graphs with $\chi_{a d}(G) \leq 2$ can be determined in polynomial time. However, for $k \geq 3$, it is NP-hard to determine whether a graph $G$ has $\chi_{a d}(G) \leq k$. It was also shown in [6] that for any positive integers $k$ and $g$, there are graphs $G$ of girth at least $g$ such that $\chi_{a d}(G)=\chi(G)=k$.

In this paper, we introduce and study a logical generalization of the adapted coloring - adapted list coloring. We will show a rather general application and derive

*Received by the editors July 25, 2007; accepted for publication (in revised form) November 9, 2007; published electronically March 7, 2008.

http://www.siam.org/journals/sidma/22-1/69838.html

${ }^{\dagger}$ Department of Mathematics, University of Illinois, Urbana, IL, 61801 and Institute of Mathematics, Novosibirsk, 630090, Russia (kostochk@math.uiuc.edu). This author's research was supported in part by NSF grant DMS-06-50784 and by grant 05-01-00816 of the Russian Foundation for Basic Research.

${ }^{\ddagger}$ Department of Applied Mathematics, National Sun Yat-Sen University, Kaohsiung, Taiwan and the National Center for Theoretical Sciences (zhu@math.nsysu.edu.tw). This author's research was partially supported by the National Science Council under grant NSC95-2115-M-110-013-MY3.

398 
several properties of such coloring.

DeFinition 1.3. Let $H$ be a multihypergraph and $F: E(H) \rightarrow \mathbf{N}$ be any edge coloring of $H$ (not required to be proper). Let a list assignment $L: V(H) \rightarrow 2^{\mathbf{N}}$ be a function that assigns a list $L(v) \subset \mathbf{N}$ to every vertex $v$ of $H$. A vertex coloring $c$ of $V(H)$ is an $L$-coloring adapted to $F$ if for every $v \in V(H), c(v) \in L(v)$, and for every color $j$, the preimage $c^{-1}(j)$ does not contain any hyperedge of color $j$. An adapted list coloring of $H$ is an $L$-coloring of $H$ adapted to $F$ for a list $L$ and an edge coloring $F$.

Adapted list coloring can model the following rather general problem. There are jobs $J_{1}, \ldots, J_{n}$ to be made and people (or machines) $P_{1}, \ldots, P_{m}$ who can perform these jobs. Some people can do several jobs, but everybody has his/her own limits. For every job $J_{i}$, there is the list $L(i)$ of people who can perform this job. For every person $P_{l}$, there are inclusion minimal sets $e_{l, 1}, \ldots, e_{l, k_{l}}$ such that $P_{l}$ cannot perform all jobs in the set $e_{l, i}$ at the same time. The question is whether we can assign to each $P_{l}$ a set $c(l)$ of jobs not containing any of the sets $e_{l, 1}, \ldots, e_{l, k_{l}}$ so that each job is assigned to somebody who can perform it. This is exactly a list adapted coloring question: Let $H$ be the hypergraph with $V(H)=\left\{J_{1}, \ldots, J_{n}\right\}$ and $E(G)=\bigcup_{l=1}^{m}\left\{e_{l, 1}, \ldots, e_{l, k_{l}}\right\}$. The people are the colors, and each vertex $J_{i}$ is given a list $L(i)$ of admissible colors. Let $F$ be the edge coloring of $H$ such that $F\left(e_{l, j}\right)=l$ for every $l=1, \ldots, m$ and $j=1, \ldots, k_{l}$. By definition, $H$ has a coloring $c$ adapted to $F$ with $c\left(J_{i}\right) \in L(i)$ exactly when there is a proper job assignment in the original problem.

There are other natural applications of adapted list coloring, but in this paper we will concentrate on the properties of this coloring.

DEFINITION 1.4. We say that a (multi)hypergraph $G$ is adaptably $k$-choosable if for every edge coloring $F$ and for every list assignment $L$ with $|L(v)|=k$ for all $v$, there exists an L-coloring of $G$ adapted to $F$. The minimum integer $k$, for which $G$ is adaptably $k$-choosable, is called the adaptable choosability of $G$ and is denoted by $\operatorname{ch}_{a d}(G)$.

It follows from the definition that if $G$ has an $L$-coloring adapted to $F$, then every subgraph $H$ of $G$ also has an $L$-coloring adapted to $F$. Let $\operatorname{ch}(G)$ denote the (ordinary) choosability of $G$. Then we have $\chi_{a d}(G) \leq \operatorname{ch}_{a d}(G) \leq \operatorname{ch}(G)$.

One may expect that for most graphs, the adaptable choosability is significantly less than the ordinary choosability. This is indeed the case. Moreover, for every multigraph $G, \operatorname{ch}_{a d}(G) \leq\lceil\sqrt{8 \Delta(G)}\rceil$. For large $\Delta(G)$ this bound is much better than the Brooks' bound for ordinary coloring. However, the situation with degeneracy is quite different. Our first main result says that there are many $d$-degenerate graphs $G$ with $\chi_{a d}(G)=d+1$. Every such graph $G$ must have $\chi(G)=\operatorname{ch}(G)=\operatorname{ch}_{a d}(G)=$ $\chi_{a d}(G)$. Furthermore, all this holds for hypergraphs.

THEOREM 1.5. For any integers $g \geq 3, r \geq 2$, and $d \geq 1$, there is an $r$-uniform hypergraph $H$ of girth at least $g$ that is d-degenerate and $\operatorname{ch}_{a d}(H)=\chi_{a d}(H)=d+1$.

Graphs $G$ with $\operatorname{ch}(G) \leq 2$ were characterized by Erdős, Rubin, and Taylor [2]. Graphs $G$ with $\chi_{a d}(G) \leq 2$ were characterized by Hell and Zhu [6]. Our second main result is a characterization of adaptably 2-choosable simple graphs. Of course, it is enough to describe adaptably 2-choosable multigraphs without vertices of degree 0 and 1.

TheOREM 1.6. A simple connected graph $G$ with minimum degree at least 2 is adaptably 2-choosable if and only if $G$ consists of two or three internally disjoint paths connecting two distinct vertices of $G$.

Theorem 1.6 yields a polynomial time algorithm that decides whether a given graph is adaptably 2 -choosable. 
We use standard notation. For a vertex $x$ of a multihypergraph $G, E_{G}(x)$ (or $E(x)$ if $G$ is clear from the context) is the set of hyperedges of $G$ containing $x$, and $d(x)=|E(x)|$ is the degree of $x$. By $\Delta(G)$ we denote the maximum degree of $G$.

For brevity, we will often say "a $(k, F)$-coloring" instead of "a vertex $k$-coloring adapted to $F$ " and "an $(L, F)$-coloring" instead of "an $L$-coloring adapted to $F$."

In the next section we give upper bounds on adaptable choosability in terms of maximum degree, in section 3 we construct many $d$-degenerate uniform hypergraphs with adaptable choosability $d+1$, and in the last section we characterize adaptably 2-choosable graphs.

2. Adaptable choosability and maximum degree. Erdős and Lovász used their famous Local Lemma to prove the following bound.

TheOREM 2.1 (see [1, Theorem 2]). A $(k+1)$-chromatic r-uniform hypergraph contains an edge which is intersected by at least $k^{r-1} / 4$ other edges. Thus, the degree of at least one vertex is $>k^{r-1} / 4 r$.

Practically repeating their proof word by word for adaptable list coloring, one gets the following result.

Proposition 2.2. If $G$ is an r-uniform (multi)hypergraph and $\Delta(G) \leq k^{r} / 4 r$, then $\operatorname{ch}_{a d}(G) \leq k$. In particular, $\operatorname{ch}_{a d}(G) \leq\lceil\sqrt{8 \Delta(G)}\rceil$ for every (multi)graph $G$.

For every fixed $r$, the order of magnitude of this bound is close to the best possible, for example, for complete $r$-uniform hypergraphs, $K_{n}^{(r)}$.

Proposition 2.3. If $n$ is large in comparison with $r$ and

$$
\Delta\left(K_{n}^{(r)}\right) \geq 2 r k^{r} \ln k,
$$

then $\operatorname{ch}_{a d}\left(K_{n}^{(r)}\right) \geq \chi_{a d}\left(K_{n}^{(r)}\right)>k$. In particular, $\operatorname{ch}_{a d}\left(K_{n}\right) \geq \chi_{a d}\left(K_{n}\right)>0.5 \sqrt{n / \ln n}$.

Proof. Let $\Delta=\Delta\left(K_{n}^{(r)}\right)$. By definition,

$$
\Delta=\left(\begin{array}{l}
n-1 \\
r-1
\end{array}\right) \leq n^{r-1} /(r-1) !
$$

Let $k$ be the largest integer satisfying (1), and let $m=\lceil n / k\rceil$. Consider a random $k$-coloring $F$ of the edges of $K_{n}^{(r)}$, where each of the edges is colored uniformly and independently from all the other edges with one of the colors $1, \ldots, k$. Let $G_{i}$ be the hypergraph formed by the edges of color $i, i=1, \ldots, k$. We will prove that

(3) with positive probability, the independence number of every $G_{i}$ is less than $\mathrm{m}$.

That would imply that for some edge $k$-coloring $F$ of $K_{n}^{(r)}$, in any vertex $k$ coloring of $K_{n}^{(r)}$ adapted to $F$, each color can appear at most $m-1$ times, and hence $K_{n}^{(r)}$ simply has no such coloring. So, we are now proving (3).

For each $i$, the probability that $G_{i}$ has an independent set of size $m$ is at most

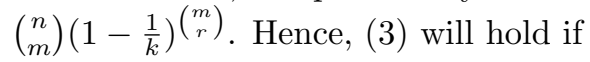

$$
\left(\begin{array}{c}
n \\
m
\end{array}\right)\left(1-\frac{1}{k}\right)^{\left(\begin{array}{c}
m \\
r
\end{array}\right)}<\frac{1}{k}
$$

Copyright $@$ by SIAM. Unauthorized reproduction of this article is prohibited. 
Since $m \geq n / k$, the left-hand side of (4) is at most

$$
\left(\frac{n e}{m}\right)^{m} \exp \left\{\frac{-1}{k}\left(\begin{array}{c}
m \\
r
\end{array}\right)\right\} \leq \exp \left\{m\left(\ln e k-\frac{1}{r k}\left(\begin{array}{c}
m-1 \\
r-1
\end{array}\right)\right)\right\} .
$$

Therefore, (4) will hold for large $n$ if

$$
0.1+\ln e k<\frac{1}{r k}\left(\begin{array}{c}
m-1 \\
r-1
\end{array}\right)
$$

By (2), for $n$ (and hence $m$ and $k$ ) large in comparison with $r$,

$$
\frac{1}{r k}\left(\begin{array}{c}
m-1 \\
r-1
\end{array}\right) \geq 0.9 \frac{1}{r k} \frac{m^{r-1}}{(r-1) !} \geq \frac{0.9}{r k} \frac{n^{r-1}}{(r-1) ! k^{r-1}} \geq \frac{0.9}{r k^{r}} \Delta .
$$

Thus (5) will hold for large $n$ if

$$
0.9 \Delta>r k^{r}(0.1+\ln e k) .
$$

By the definition of $k$ for large $n, 0.1+\ln e k<1.1 \ln k$. Hence, to satisfy (6), it is enough that $0.9 \Delta>1.1 r k^{r} \ln k$, which follows from (1).

For a positive integer $k$, let $f(k)$ (respectively, $f_{m}(k)$ ) be the largest $\Delta$ such that $\operatorname{ch}_{a d}(G) \leq k$ for every simple graph (respectively, multigraph) $G$ with maximum degree at most $\Delta$. It follows from Propositions 2.2 and 2.3 that $k^{2} / 8 \leq f_{m}(k) \leq$ $f(k) \leq 4 k^{2} \ln k$. It is also interesting to find better estimates of $f_{m}(k)$ and $f(k)$ for small $k$. Theorem 1.6 yields $f_{m}(2)=f(2)=2$. Theorem 2.4 below provides another upper bound for $\operatorname{ch}_{a d}(G)$ when $k$ is small.

THEOREM 2.4. If $G$ is a multigraph with maximum degree $\Delta$, then $\operatorname{ch}_{a d}(G) \leq$ $\lceil\Delta / 2\rceil+1$.

Proof. Let $F$ be an arbitrary edge coloring of $G$. We shall prove that if each vertex $x$ of $G$ is given a list $L(x)$ of at least $\lceil d(x) / 2\rceil+1$ permissible colors, then $G$ has an $(L, F)$-coloring. (Recall that " $(L, F)$-coloring" means " $L$-coloring adapted to $F "$ ") We prove this by induction on the number of edges of $G$. Without loss of generality, we assume that $G$ is connected. If $G$ has a vertex of degree 1 , then $|L(x)| \geq 2$. Therefore, any $(L, F)$-coloring of $G-x$ can be extended to an $(L, F)$-coloring of $G$. If $G$ is a cycle, then the conclusion follows from Theorem 1.6 in section 4 . If $G$ has a vertex $x$ of degree 3, then $|L(x)| \geq 3$. Assume $e \in E(x)$ and $F(e)=j$. Remove color $j$ from $L(x)$ to obtain a list assignment $L^{\prime}$ of $G-e$. By induction hypothesis, $G-e$ has an $\left(L^{\prime}, F\right)$-coloring $c$. Since $c(x) \neq F(e), c$ is also an $(L, F)$-coloring of $G$. Assume that $G$ has a vertex $x$ of degree $d(x) \geq 4$ with $|L(x)|=\lceil d(x) / 2\rceil+1<d(x)$. If for some edge $e \in E(x), F(e) \notin L(x)$, then an $(L, F)$-coloring of $G-e$ is also an $(L, F)$-coloring of $G$. Thus we assume that $F(e) \in L(x)$ for all $e \in E(x)$. Then there are two edges $e, e^{\prime} \in E(x)$ with $F(e)=F\left(e^{\prime}\right)=j$. Remove color $j$ from $L(x)$ to obtain a list assignment $L^{\prime}$ of $G-\left\{e, e^{\prime}\right\}$. By induction hypothesis, $G-\left\{e, e^{\prime}\right\}$ has an $\left(L^{\prime}, F\right)$-coloring $c$. Since $c(x) \neq F(e)=F\left(e^{\prime}\right), c$ is also an $(L, F)$-coloring of $G$.

Theorem 2.4 implies that $f_{m}(3) \geq 4$. Figure 1 shows an edge 3 -colored multigraph $G$ with $\Delta(G)=5$ that has no vertex 3 -coloring adaptable to the given edge coloring. Indeed, assume it has such a 3 -coloring $c$. Then either $c(v) \neq 1$ or $c\left(v^{\prime}\right) \neq 1$. By the left-right symmetry, we may assume that $c(v) \neq 1$. Now by the up-down symmetry, we may assume that $c(v)=2$. Then $c(x) \neq 2$ and $c(y) \neq 2$. Since edge $x y$ has color 1 , at least one of $x$ and $y$ (say, $x$ ) should be colored with 3 . Thus all three vertices 


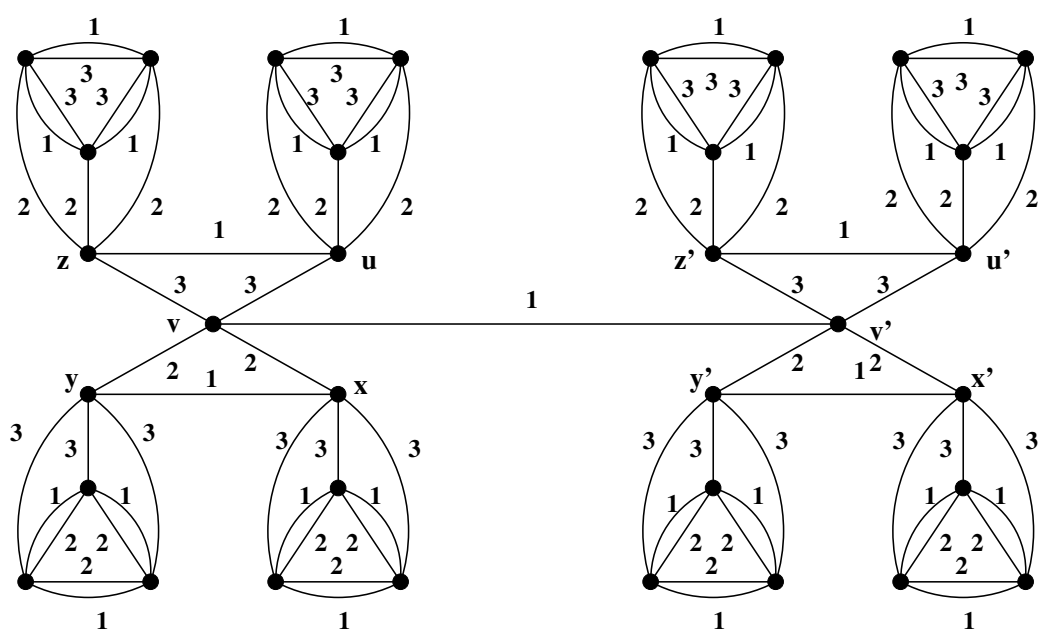

FIG. 1. A multigraph $G$ with $\operatorname{ch}_{a d}(G) \geq 4$.

below $x$ can be colored with only 1 or 2 , but the double edges between these vertices forbid any two of them to have the same color.

This example shows that $f_{m}(3)=4$. We also have an example showing that $f(3) \leq 5$, but were not able to determine whether $f(3)$ is 4 or 5 .

3. Adaptable choosability of $\boldsymbol{d}$-degenerate graphs. While the upper bounds on adaptable choosability of graphs in terms of maximum degree are significantly better than those on ordinary choosability, this is not the case for bounds in terms of degeneracy. Recall that a multihypergraph $G$ is $d$-degenerate if each subhypergraph $G^{\prime}$ of $G$ has a vertex of degree at most $d$. It is obvious that $\operatorname{ch}(G) \leq d+1$ for every $d$-degenerate $G$. Since $\operatorname{ch}_{a d}(G) \leq \operatorname{ch}(G)$, we have $\operatorname{ch}_{a d}(G) \leq d+1$. In [6], examples of $d$-degenerate graphs $G$ with $\chi_{a d}(G)=d+1$ are given. It is also shown in [6] that for any positive integer $d$, there are graphs of large girth with $\chi_{a d}(G)=\chi(G)=d+1$. However, these graphs are far from being $d$-degenerate. In this section, we prove Theorem 1.5, which is a common strengthening and generalization of these results in [6]. But first we prove a weaker statement.

Proposition 3.1. For any integers $g \geq 3, r \geq 2$, and $k \geq 1$, there is an $r$-uniform hypergraph $G$ with girth at least $g$ and $\operatorname{ch}_{a d}(G) \geq k$.

Proof. Let $n$ be large in comparison with $g, r$, and $k$. Consider the random $r$-uniform $n$-vertex hypergraph $\mathbf{H}(n, r, k)$ with colored edges (and labeled vertices) obtained in the following way: Every $r$-element subset $e$ of $\{1, \ldots, n\}$ independently of all other such $r$-tuples with probability $1-(\ln \ln n) / n^{r-1}$ is not in $\mathbf{H}(n, r, k)$, and for each $i=1, \ldots, k$, with probability $\ln \ln n / k n^{r-1}, e$ is an edge in $\mathbf{H}(n, r, k)$ colored with color $i$.

Let $F$ denote the obtained edge coloring, and let $H_{i}$ be the subhypergraph of $\mathbf{H}(n, r, k)$ induced by the edges of color $i, i=1, \ldots, k$. We will prove using a standard argument that with positive probability both (i) and (ii) below hold:

(i) there is a set $S$ of at most $n / 3$ vertices such that $\mathbf{H}(n, r, k)-S$ has no cycles of length $g$ or less;

(ii) independence number of every $H_{i}$ is at most $n / 2 k$.

If we prove this, then there is a hypergraph $H^{\prime}$ for which both (i) and (ii) hold. Hence, the hypergraph $H^{\prime \prime}=H^{\prime}-S$ has at least $2 n / 3$ vertices and in any $(k, F)$ - 
coloring of $H^{\prime \prime}$ each color can be used for at most $n / 2 k$ vertices. Thus $H^{\prime \prime}$ has no such coloring.

First, consider property (i). For given two vertices $v$ and $w$, the probability that $\mathbf{H}(n, r, k)$ has at least two edges containing both $v$ and $w$ is at most

$$
\left.\sum_{t=2}^{\left(\begin{array}{c}
n-2 \\
r-2
\end{array}\right)}\left(\frac{\ln \ln n}{n^{r-1}}\right)^{t}\left(\begin{array}{c}
n-2 \\
r-2 \\
t
\end{array}\right)\right) \leq \sum_{t=2}^{\left(\begin{array}{c}
n-2 \\
r-2
\end{array}\right)} \frac{1}{t !}\left(\frac{\ln \ln n}{(r-2) ! n}\right)^{t} \leq\left(\frac{\ln \ln n}{n}\right)^{2} .
$$

Since there are $\left(\begin{array}{l}n \\ 2\end{array}\right)$ such pairs $\{v, w\}$, the expectation $E_{2}$ of the number of the cycles of length two in $\mathbf{H}(n, r, k)$ is at most $0.5(\ln \ln n)^{2}$. Similarly, for $3 \leq j \leq g$, the expectation $E_{j}$ of the number of the cycles of length $j$ in $\mathbf{H}(n, r, k)$ is at most $(\ln \ln n)^{j} / j$. Therefore, the expectation $E_{0}$ of the number of the cycles of all lengths from 2 to $g$ in $\mathbf{H}(n, r, k)$ is at most $(\ln \ln n)^{g}$. Hence for $n$ large, (i) holds with probability at least $1-3(\ln \ln n)^{g} / n>0.9$.

Similar to the proof of Proposition 2.3, for each $1 \leq i \leq k$, the probability that $H_{i}$ has an independent set of size $m=\lceil n / 2 k\rceil$ is at most

$$
\left(\begin{array}{c}
n \\
m
\end{array}\right)\left(1-\frac{\ln \ln n}{k n^{r-1}}\right)^{\left(\begin{array}{c}
m \\
r
\end{array}\right)} \leq\left(\frac{n e}{m} \exp \left\{-\frac{\ln \ln n}{k n^{r-1}}\left(\begin{array}{c}
m-1 \\
r-1
\end{array}\right) / r\right\}\right)^{m} .
$$

For fixed $r$ and $k$ and large $n$, the $m$ th root of the right-hand side is at most

$$
2 k e \exp \left\{-0.5 \frac{\ln \ln n}{k n^{r-1}} \frac{n^{r-1}}{r ! k^{r-1}}\right\} \leq 6 k \frac{(k r)^{r}}{\ln n}<\frac{1}{2 k} .
$$

It follows that with probability at least $0.9-\frac{k}{2 k}=0.4$, both (i) and (ii) hold for $\mathbf{H}(n, r, k)$.

Now we are ready to prove Theorem 1.5. For convenience, we restate it here.

TheOREM 1.5. For any integers $g \geq 3, r \geq 2$, and $d \geq 1$, there is an $r$-uniform $d$-degenerate hypergraph $G$ with girth at least $g$ and $\operatorname{ch}_{a d}(G)=\chi_{a d}(G)=d+1$.

Proof. As observed above, $\chi_{a d}(G) \leq \operatorname{ch}_{a d}(G) \leq \operatorname{ch}(G) \leq d+1$ for every $d$ degenerate $G$. So, it suffices to show that there is an $r$-uniform $d$-degenerate hypergraph $G^{\prime}$ of girth at least $g$ with $\chi_{a d}\left(G^{\prime}\right)>d$, i.e., $G^{\prime}$ has a edge $d$-coloring $F^{\prime}$ such that $G^{\prime}$ has no vertex $\left(d, F^{\prime}\right)$-coloring.

We prove this by induction on $d$. When $d=1$, for each $r$, let $G_{r}$ be the hypergraph that contains one edge of size $r$ and nothing else. Clearly, $\chi_{a d}\left(G_{r}\right)=2$.

Assume that the conclusion is true for degeneracy $d-1$ and all $r$ and $g$. Given positive integers $r$ and $g$, let $H$ be an $r$-uniform $(d-1)$-degenerate hypergraph of girth at least $g$ with $\chi_{a d}(H)=d$. Let $n=|V(H)|$ and $r^{\prime}=n(r-1)$. By Proposition 3.1, there exists an $r^{\prime}$-uniform hypergraph $G=(V, E)$ of girth at least $g$ with $\chi_{a d}(G) \geq d+$ 1. Let $G^{\prime}$ be the hypergraph constructed as follows. For each edge $s=\left\{v_{1}, v_{2}, \ldots, v_{r^{\prime}}\right\}$ of $G$, add a disjoint copy of $H$, called $H_{s}$ (including the vertices and edges). The vertices of $H_{s}$ are $\left\{u_{1, s}, u_{2, s}, \ldots, u_{n, s}\right\}$. Partition these vertices in $s$ into $n$ parts, $X_{1}, X_{2}, \ldots, X_{n}$, each of cardinality $r-1$. Add the edges $\left\{v_{j, s}\right\} \cup X_{j}(j=1,2, \ldots, n)$. We denote the subhypergraph of $G^{\prime}$ induced by $s \cup V\left(H_{s}\right)$ by $G_{s}$ (see Figure 2).

Since $\chi_{a d}(H)=d$, there is a $(d-1)$-edge coloring $F$ of $H$ such that $H$ has no $(d-1, F)$-coloring. Since $\chi_{a d}(G) \geq d+1$, there is an edge $d$-coloring $Q$ of $G$ such that $G$ has no $(d, Q)$-coloring.

Define edge $d$-coloring $F^{\prime}$ of $G^{\prime}$ as follows. Let $e$ be a hyperedge of $G^{\prime}$. Then $e$ is contained in $G_{s}$ for some hyperedge $s$ of $G$. Assume that $Q(s)=j$. If $e$ is a hyperedge 


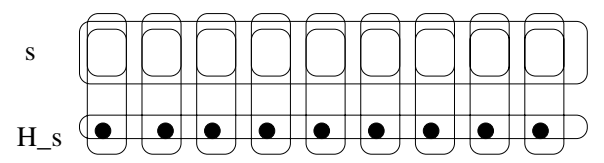

FIG. 2. The part of $G^{\prime}$ corresponding to an edge $s$ of $G$.

of $H_{s}$, then let

$$
F^{\prime}(e)= \begin{cases}F(e) & \text { if } F(e) \neq j \\ d & \text { if } F(e)=j\end{cases}
$$

If $e$ is of the form $\left\{v_{i, e}\right\} \cup X_{i}$, then let $F^{\prime}(e)=i$. We shall show that $G^{\prime}$ has no $\left(d, F^{\prime}\right)$-coloring. Assume to the contrary that $c^{\prime}: V\left(G^{\prime}\right) \rightarrow\{1,2, \ldots, d\}$ is such a coloring. Since $\chi_{a d}(G)=d+1$, there is a hyperedge $s=\left\{v_{1}, v_{2}, \ldots, v_{r^{\prime}}\right\}$ of $G$ such that $c^{\prime}\left(v_{1}\right)=c^{\prime}\left(v_{2}\right)=\cdots=c^{\prime}\left(v_{r^{\prime}}\right)=Q(s)$. If $c\left(v_{i, s}\right)=Q(s)$ for any $i \in\{1,2, \ldots, n\}$, then $\left\{v_{i, s}\right\} \cup X_{i}$ is a hyperedge of $G^{\prime}$ of color $Q(s)$, and all the vertices of this hyperedge are colored by $Q(s)$, which is a contradiction. If $c^{\prime}\left(v_{i, s}\right) \neq Q(s)$ for all $i \in\{1,2, \ldots, n\}$, then the restriction of $c^{\prime}$ to the copy $G_{s}$ of $G$ is a $(d-1, F)$-coloring, contrary to our assumption.

By construction, $G^{\prime}$ is $d$-degenerate and has girth at least $g$. This proves Theorem 1.5.

4. Adaptably 2-choosable graphs. The next observation will be quite helpful.

Proposition 4.1. Let $G$ be a multihypergraph, $L$ be a list assignment for the vertices of $G$, and $F$ be an edge coloring of $G$. If $G$ has no $(L, F)$-coloring, but after deleting any edge or vertex the resulting multihypergraph $G^{\prime}$ has, then

(a) for every $e \in E(G)$, color $F(e)$ is present in the list of every $v \in e$;

(b) for every $v \in V(G)$, every color in $L(v)$ is a color of some edge containing $v$.

Proof. If $F(e)$ is not present in the list of some vertex $v \in e$ and $G-e$ has an $(L, F)$-coloring, then this coloring is also an $(L, F)$-coloring of $G$. If $L(v)$ contains a color $\alpha$ that is not a color of any edge containing $v$, and $G-v$ has an $(L, F)$-coloring $c$, then letting $c(v)=\alpha$ we get an $(L, F)$-coloring of $G$.

Given a coloring $F$ of the edges of a hypergraph $G$, there is a unique list assignment $L$ such that both (a) and (b) in Proposition 4.1 hold. We will denote this list assignment by $L_{G, F}$ and will say that it respects $F$. Furthermore, instead of " $\left(L_{G, F}, F\right)$-coloring of $G$ " we will simply say " $F$-coloring of $G$." For a vertex $x$ of $G$, let $F(x)=\{F(e): e \in E(x)\}$.

Lemma 4.2. Let $P=\left(v_{0}, v_{1}, \ldots, v_{n}\right)$ be a path and $F$ be any edge coloring of $P$. Then $P$ has no $F$-coloring.

Proof. Let $L=L_{P, F}$. For $i=1, \ldots, n$, let $e_{i}=v_{i-1} v_{i}$. Assume that $c$ is an $F$-coloring of $P$. Then $L\left(v_{0}\right)=\left\{F\left(e_{1}\right)\right\}$ and hence $c\left(v_{0}\right)=F\left(e_{1}\right)$. This forces $L\left(v_{1}\right) \neq\left\{F\left(e_{1}\right)\right\}$. Since $L$ respects $F$, we must have $F\left(e_{2}\right) \neq F\left(e_{1}\right)$ and $c\left(v_{1}\right)=F\left(e_{2}\right)$. Repeating this argument, we conclude that $c\left(v_{i}\right)=F\left(e_{i+1}\right)$ for $i=0,1, \ldots, n-1$. But $L\left(v_{n}\right)=\left\{F\left(e_{n}\right)\right\}$ and hence $c\left(v_{n}\right)=F\left(e_{n}\right)=c\left(v_{n-1}\right)$, contrary to our assumption that $c$ is an $F$-coloring of $P$.

Corollary 4.3. Let $C$ be a cycle and $F$ be an edge coloring of $P$. If for some vertex $x \in V(C),\left|L_{C, F}(x)\right|=1$, then $C$ has no $F$-coloring.

Proof. Let $L=L_{C, F}$. Split the vertex $x$ of $C$ into two vertices $x^{\prime}, x^{\prime \prime}$ with each of $x^{\prime}, x^{\prime \prime}$ incident to one edge in $E(x)$, and let $L\left(x^{\prime}\right)=L\left(x^{\prime \prime}\right)=L(x)$. The resulting 
graph is a path $P$, and $F$ can be viewed as an edge coloring of $P$. Any $F$-coloring of $C$ is also an $F$-coloring of $P$. Thus the conclusion follows from Lemma 4.2.

LEMMA 4.4. If $G$ is a subdivision of $K_{4}$, then $G$ is not adaptably 2-choosable.

Proof. Consider first the case that no two adjacent edges are subdivided, i.e., that the subdivided edges form a matching of $K_{4}$. Then the set of nonsubdivided edges of $K_{4}$ contains a 4 -cycle $C_{4}$. Let $F$ be an edge coloring of $G$ such that the edges of this 4 -cycle are colored by color 1 . The other edges of $G$ are colored arbitrarily so that each vertex is incident to edges of two distinct colors. For example, the first two graphs in Figure 3 are colored in this way. Let $L=L_{G, F}$. Assume that $G$ has an $F$-coloring $c$.

Let the four vertices of the 4 -cycle $C_{4}$ be $v_{1}, v_{2}, v_{3}, v_{4}$ (these four vertices are also the only vertices of $G$ of degree 3 ) in this order. Then either neither of $v_{1}$ and $v_{3}$ is colored by color 1 , or neither of $v_{2}$ and $v_{4}$ is colored by 1 . Without loss of generality, assume that neither of $v_{1}$ and $v_{3}$ is colored by 1 . Then the restriction of $c$ to the path $P_{1,3}$ of $G$ connecting $v_{1}$ and $v_{3}$ is an $\left(L^{\prime}, F\right)$-coloring of $P_{1,3}$, where $L^{\prime}=L_{P_{1,3}, F}$. This contradicts Lemma 4.2 .

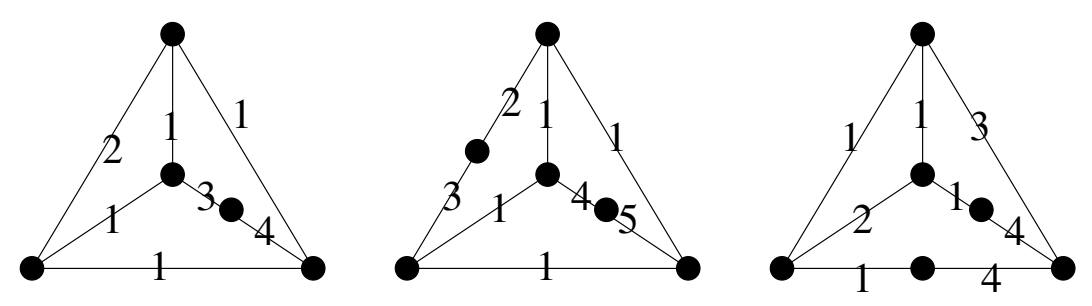

FIG. 3. Some subdivisions of $K_{4}$.

Next, assume that $G$ has two adjacent edges that are subdivided. Let $H$ be the third graph in Figure 3. Then $G$ is a subdivision of $H$.

Let $F$ be an edge coloring $F$ of a multigraph $Q$ and $L=L_{G, F}$. We say that $F$ is difficult if the following hold:

(i) $|L(v)|=2$ for each $v \in V(Q)$.

(ii) For each color $j$, the edges of color $j$ induce a tree $T_{j}$.

(iii) $Q$ has no $(L, F)$-coloring.

The edge coloring $F$ of graph $H$ in Figure 3 is difficult: (i) and (ii) are obvious. To see that (iii) holds, observe that if the top vertex is colored by 1 , then the vertex at the center and the leftmost vertex must both be colored by 2 , which is a contradiction. If the top vertex is colored by 3 , then the rightmost vertex must be colored by 4 , which forces both degree 2 vertices of $H$ be colored by 1, which in turn forces the vertex at the center and the leftmost vertex be both colored by 2 ; which is again a contradiction.

CLAIM 4.1. Suppose that a multigraph $Q$ has a difficult coloring and $Q^{\prime}$ is obtained from $Q$ by subdividing one edge. Then $Q^{\prime}$ has a difficult coloring.

Proof. Assume $Q^{\prime}$ is obtained from $Q$ by inserting a vertex $w$ on an edge $e^{*}=u v$ of $Q$. In other words, $e^{*}$ is subdivided into two edges $e^{\prime}=u w$ and $e^{\prime \prime}=w v$. Assume that $e^{*}$ has color $j$. Let $T_{j}$ be the tree induced by the edges of color $j$. Let $T_{j}^{\prime}$ be the tree obtained from $T_{j}$ by subdividing the edge $e^{*}$ into $e^{\prime}=w u$ and $e^{\prime \prime}=w v$. Vertex $w$ separates $E\left(T_{j}^{\prime}\right)$ into two parts, say $E_{1}$ (containing $e^{\prime}$ ) and $E_{2}$ (containing $e^{\prime \prime}$ ). Let $j^{\prime}$ be a new color (a color not used on any edge of $Q$ ). Let $F^{\prime}$ be the edge coloring of 
$Q^{\prime}$ defined as follows:

$$
F^{\prime}(e)= \begin{cases}j & \text { if } e \in E_{1}, \\ j^{\prime} & \text { if } e \in E_{2}, \\ F(e) & \text { otherwise }\end{cases}
$$

Now we show that $F^{\prime}$ is a difficult coloring of $Q^{\prime}$. It is obvious that (i) and (ii) hold. Assume to the contrary that $c^{\prime}$ is an $L_{Q^{\prime}, F^{\prime}}$-coloring of $Q^{\prime}$ adaptable to $F^{\prime}$. Let $c$ be the $L$-coloring of $Q$ defined as

$$
c(x)= \begin{cases}j & \text { if } c^{\prime}(x)=j \text { or } j^{\prime}, \\ c^{\prime}(x) & \text { otherwise. }\end{cases}
$$

Observe that $c^{\prime}(w) \in\left\{j, j^{\prime}\right\}$, and hence either $c^{\prime}(u) \neq j$ or $c^{\prime}(v) \neq j^{\prime}$. In either case, the ends $u$ and $v$ of the edge $e^{*}$ cannot both have color $j$. This implies that $c$ is an $(L, F)$-coloring of $Q$, contrary to our assumption that $Q$ is not $F$-colorable.

By repeatedly applying Claim 4.1, we derive that each subdivision of $H$ has a difficult edge coloring, and hence is not adaptably 2-choosable. This completes the proof of Lemma 4.4.

It follows from Lemma 4.4 that adaptably 2 -choosable graphs are $K_{4}$-minor free, i.e., series-parallel graphs.

LEMMA 4.5. If a connected multigraph $G$ has two cycles of length at least 3 that have at most one vertex in common, then $G$ is not adaptably 2-choosable.

Proof. Assume $G$ has two cycles $C_{1}$ and $C_{2}$ of length at least 3 that have at most one vertex in common. Let $P$ be a shortest path connecting $C_{1}$ and $C_{2}$. Let $\{v\}=V(P) \cap V\left(C_{1}\right)$ and $\left\{v^{\prime}\right\}=V(P) \cap V\left(C_{2}\right)$. This includes the case that $v^{\prime}=v$.

Let $G^{\prime}$ be the subgraph of $G$ induced by $V\left(C_{1} \cup C_{2} \cup P\right)$. Let $F$ be an edge coloring of $G^{\prime}$ such that (1) the two edges of $C_{1}$ incident with $v$ are colored with 1 , (2) the two edges of $C_{2}$ incident with $v^{\prime}$ are colored with 2, and (3) the remaining edges are colored so that each vertex is incident to edges of two distinct colors (for example, all other edges may have different colors). We shall show that there is no $F$-coloring of $G^{\prime}$. Assume to the contrary that $c$ is such a coloring. If $c(v)=1$, then the restriction of $c$ to $C_{1}$ is an $F$-coloring of $C_{1}$, contrary to Corollary 4.3. Similarly, if $c\left(v^{\prime}\right)=2$, then the restriction of $c$ to $C_{2}$ is an $F$-coloring of $C_{2}$. If $c(v) \neq 1$ and $c\left(v^{\prime}\right) \neq 2$, then the restriction of $c$ to the path $P$ is an $F$-coloring of $P$, a contradiction to Lemma 4.2 .

LEMMA 4.6. If a simple graph $G$ consists of four internally vertex-disjoint paths $P_{1}, P_{2}, P_{3}$, and $P_{4}$ connecting two vertices $u, v$, then $G$ is not adaptably 2-choosable.

Proof. If $P$ is a path not of length 2, then there is a proper edge coloring of $P$ with at most three colors, such that the two end edges (which can be the same edge if $P$ has length 1) are colored with the same color. If $P$ is a path not of length 1 , then there is a proper edge coloring of $P$ with at most three colors, such that the two end edges are colored with two given distinct colors.

Case 1. At least two of the paths are not of length 2. Since at most one of $P_{1}, \ldots, P_{4}$ has length 1 , we can renumber our paths so that $P_{1}$ and $P_{2}$ are not of length 1 , and $P_{3}$ and $P_{4}$ are not of length 2. Color properly the edges of $P_{1}$ (respectively, $P_{2}$ ) so that the edge incident to $u$ is colored by 1 (respectively, by 2 ), and the edge incident to $v$ is colored by 2 (respectively, by 1 ). Color the edges of $P_{3}$ (respectively, $P_{4}$ ) so that both end edges are colored by 1 (respectively, by 2 ). We have $L(u)=L(v)=\{1,2\}$. If $c$ is an $F$-coloring of $G$, then $c(u), c(v) \in\{1,2\}$. If $c(u)=c(v)=1$, then the restriction of $c$ to $P_{3}$ is an $F$-coloring of $P_{3}$, contrary to Lemma 4.2. If $c(u)=c(v)=2$, then we 
get a contradiction by considering path $P_{4}$. If $c(u)=1$ and $c(v)=2$, then we obtain a contradiction with the edge coloring of $P_{1}$, and if $c(u)=2$ and $c(v)=1$, then we obtain a contradiction with the edge coloring of $P_{2}$.

Case 2. Each of $P_{1}, P_{2}$, and $P_{3}$ has length 2 and the length of $P_{4}$ is not 1. By above we can color the edges of $G$ so that (a) the edges incident to $u$ in $P_{1}$ and $P_{2}$ are colored with 1 , and in $P_{3}$ and $P_{4}$ are colored with 2 ; (b) the edges incident to $v$ in $P_{1}$ and $P_{3}$ are colored with 3 , and in $P_{2}$ and $P_{4}$ are colored with 4 . Now $L(u)=\{1,2\}$ and $L(v)=\{3,4\}$. Similarly to Case 1, each of the four paths forbids one of the four possibilities $(1,3),(1,4),(2,3)$, and $(2,4)$ for the pair $(c(u), c(v))$.

Case 3. $\left|E\left(P_{1}\right)\right|=\left|E\left(P_{2}\right)\right|=\left|E\left(P_{3}\right)\right|=2$ and $\left|E\left(P_{4}\right)\right|=1$. For $1 \leq i \leq 3$, let $w_{i}$ be the central vertex of the path $P_{i}$. We let $F(u v)=F\left(u w_{1}\right)=F\left(w_{2} v\right)=1$, $F\left(u w_{2}\right)=F\left(u w_{3}\right)=2$ and $F\left(w_{1} v\right)=F\left(w_{3} v\right)=3$. It is easy to check that $G$ has no $F$-coloring for this $F$.

Now we are ready to prove Theorem 1.6. For convenience, we restate it here.

TheOREM 1.6. A simple connected graph $G$ with minimum degree at least 2 is adaptably 2-choosable if and only if $G$ consists of two or three internally disjoint paths connecting two distinct vertices of $G$.

Proof. Assume first that a simple connected graph $G$ with minimum degree at least 2 is adaptably 2-choosable. By Lemma $4.4 G$ is series-parallel. Furthermore, by Lemma 4.5, $G$ is 2-connected and any two cycles of $G$ have at least two vertices in common. This implies that $G$ has two vertices $u$ and $v$ such that for some $k \geq 2$, $G$ consists of $k$ internally vertex disjoint paths connecting $u$ and $v$. By Lemma 4.6, $k \leq 3$. This proves the "only if" part of the theorem.

Suppose now that the "if" part of the theorem does not hold. Then there is an inclusion minimal subgraph $G$ of a graph $G^{\prime}$ consisting of three internally disjoint paths connecting some vertices $u$ and $v$ that has no $L$-coloring adapted to an edgecoloring $F$, where $|L(w)|=2$ for every $w \in V(G)$. By Proposition 4.1, $G$ has no isolated or pendant vertices, and it is enough to consider $F$-colorings.

Let $F$ be an edge coloring of $G$ such that $|F(x)|=2$ for each vertex $x$ of $G$. Assume $F(u)=\{a, b\}$ and $F(v)=\{c, d\}$ (where $\{a, b\}$ and $\{c, d\}$ need not be disjoint).

There are four distinct ways to color $u$ and $v$, namely $(c(u), c(v)) \in\{(a, c),(a, d)$, $(b, c),(b, d)\}$. For each $P_{i}$, there is exactly one choice of the pair $(c(u), c(v))$ that cannot be extended to a coloring of $P_{i}$ adapted to $F$. Thus, if $2 \leq k \leq 3$, then at least one of the four choices of $(c(u), c(v))$ can be extended to a coloring adapted to $F$ for each of the $u, v$-paths.

Remark. Using the proof of the "only if" part of the theorem, one can characterize also all adaptably 2-choosable multigraphs. The remaining argument is a simple case analysis, so we just give a preliminary idea and state the characterization.

We need the following notion: a double $x, y$-path is a multigraph whose underlying simple graph is an $x, y$-path and in which every edge has multiplicity two. If $G$ has a vertex $x$ with only one neighbor $y$ (does not matter how many edges connect $x$ and $y$ ) and $G-x$ is adaptably 2 -choosable, then $G$ is also adaptably 2 -choosable. Thus it is enough to characterize adaptably 2-choosable multigraphs whose underlying simple graphs have minimum degree at least two. Theorem 1.6 tells us that these underlying graphs have very limited structure.

TheOREM 4.7. A connected multigraph $G$ whose underlying simple graph $G_{s}$ has minimum degree at least 2 is adaptably 2-choosable if and only if all of the following holds:

(a) $G_{s}$ consists of two or three internally disjoint paths connecting two distinct vertices $x$ and $y$ of $G$;

Copyright $@$ ( ) by SIAM. Unauthorized reproduction of this article is prohibited. 
(b) all double $x, y$-paths have the same parity;

(c) if there are three internally disjoint $x, y$-paths $P_{1}, P_{2}$, and $P_{3}$ such that $P_{1}$ is a double $x, y$-path of an odd length, then one of $P_{2}$ and $P_{3}$ has length 1 ;

(d) if there are three internally disjoint $x, y$-paths $P_{1}, P_{2}$, and $P_{3}$ such that $P_{1}$ is a double $x, y$-path of an even length, then at least one of $P_{2}$ and $P_{3}$ has length 2 .

This description is somewhat more sophisticated than Theorem 1.6, but it yields a polynomial-time algorithm for recognition of adaptably 2-choosable multigraphs.

Acknowledgment. We thank a referee for helpful comments.

\section{REFERENCES}

[1] P. ERdős ANd L. LovÁsz, Problems and results on 3-chromatic hypergraphs and some related questions, in Infinite and Finite Sets, A. Hajnal et. al., eds., Colloq. Math. Soc. János Bolyai 10, North-Holland, Amsterdam, 1975, pp. 609-627.

[2] P. Erdős, A. Rubin, And H. TAylor, Choosability in graphs, in Proceedings of the West Coast Conference on Combinatorial Graph Theory and Computing, Arcata, CA, 1979, Congress. Numer., 26 1979, Utilitas Math. Winnipeg, Manitoba, 1980, pp. 125-157.

[3] T. Feder and P. Hell, Full constraint satisfaction problems, SIAM J. Comput., 36 (2006), pp. 230-246.

[4] T. Feder, P. Hell, S. Klein, and R. Motwani, List partitions, SiAM J. Discrete Math., 16 (2003), pp. 449-478.

[5] T. Feder, P. Hell, D. Kral, and J. Sgall, Two algorithms for general list matrix partition, in Proceedings of the 16th Annual ACM-SIAM Symposium on Discrete Algorithms (SODA), Vancouver, BC, 2005, pp. 870-876.

[6] P. Hell And X. Zhu, Adaptable chromatic number of graphs, European J. Combin., to appear.

[7] P. Hell and J. NeŠEtřIl, Graphs and homomorphisms, Oxford University Press, Oxford, UK, 2004.

Copyright (c) by SIAM. Unauthorized reproduction of this article is prohibited. 